\title{
CONF-951155--84-5umm - ENERGETIC ION BEAMS IN SEMICONDUCTOR PROCESSING: SUMMARY OF A DOE PANEL STUDY SAND95. $453 \mathrm{C}$
}

S. T. PICRAUX, E. CHASON, Sandia National Laboratories, Albuquerque, NM; J. M. POATE, AT\&T Bell Laboratories, Murray Hill, NJ; J. O. BORLAND, Genus Corporation, Newburyport, MA; M. I. CURRENT, Applied Materials, Austin, TX; T. DIAZ DE LA RUBIA, Lawrence Livermore National Laboratory, Livermore, CA; D. J. EAGLESHAM, AT\&T Bell Laboratories, Murray Hill, NJ; O. W. HOLLAND, Oak Ridge National Laboratory, Oak Ridge, TN; M. E. LAW, University of Florida, Gainesville, FL; C. W. MAGEE, Evans East, Plainsboro
MAYER, Arizona State University, Tempe, AZ; J. MELNGAILIS, University of Maryland, College Park, MD; and A. F. TASCH, University of Texas, Austin, TX.

MAR 151996

OSTI

\section{ABSTRACT}

The trend toward smaller dimensions in integrated circuit technology presents severe physical and engineering challenges for ion implantation. These challenges, together with the need for physically-based models at exceedingly small dimensions, are leading to a new level of understanding of fundamental defect science in silicon. Recently the DOE Council on Materials requested that our panel examine the current status and future research opportunities in the area of ion beams in semiconductor processing. Particularly interesting are the emerging approaches to defect and dopant distribution modeling, transient enhanced diffusion, high energy implantation and defect accumulation, and metal impurity gettering. These topics were explored both from the perspective of the emerging science issues and the technology challenges.

\section{INTRODUCTIONं}

Ion implantation is one of the most important processing tools in Si integrated circuit technology. However, in the move to ever finer dimensions real physical and engineering limits are being confronted (see Table I for the Semiconductor Industry Association projections for future device dimensions). For example, in low energy implantation for shallow junction formation, understanding and controlling defect enhanced diffusion are becoming critical factors in building in the needed dimensional control for circuit design. A panel study commissioned by the U.S. Department of Energy Council on Materials addressed these and other fundamental science and technology issues confronting the semiconductor processing industry in the area of ion beam processing [2]. We briefly summarize those results here.

Foremost among the issues examined are the atomic level understanding of the physical phenomena and the incorporation of this understanding into realistic computer-based models. Coupled with these critical needs in the understanding of the basic processes are the challenges for future commercial machine development in the very low energy and the high energy regimes. In addition, focused ion beams are becoming increasingly important, for example in mask repair, while their role in implantation and lithography is still uncertain.

In Fig. 1 we illustrate many of the key steps where ion implantation is used in the manufacturing of complementary metal oxide semiconductor (CMOS) technology. Typically 15 or more implantations will be carried out in the course of some 300 or more steps in the 
fabrication of an integrated circuit. Low fluence implants $\left(10^{12} / \mathrm{cm}^{2}\right)$ are used for the threshold adjust of the channel, high energy implants ( $300 \mathrm{keV}$ to $1 \mathrm{MeV}$ ) are used to create $n$ and $\mathrm{p}$ type wells (tubs) for the complementary MOS transistors and for enhanced performance (antipunchthrough and retrograde well profiles), low energy implants (10 to $40 \mathrm{keV}$ ) are used for the source and drain steps, guard-ring implants provide isolation between adjacent regions of the circuit, various other isolating implants may be used below the transistor channels to enhance performance, and high fluence implants $\left(10^{15} / \mathrm{cm}^{2}\right)$ are use for doping the polycrystalline Si gate. Of these the low energy source and drain implants along with the high energy implants present the greatest challenges and lead to the most interesting research questions.

Table I. Projected MOS source/drain doping technology requirements from the SIA roadmap [1].

\begin{tabular}{||l|c|c|c|c|c|c||}
\hline First Year of Production & 1995 & 1998 & 2001 & 2004 & 2007 & 2010 \\
\hline $\begin{array}{l}\mathrm{L}_{\mathrm{i}}-\text { Transistor gate length } \\
(\mu \mathrm{m})\end{array}$ & 0.35 & 0.25 & 0.18 & 0.13 & 0.10 & 0.07 \\
\hline $\begin{array}{l}\mathrm{Xj}-\text { Junction depth at chan. } \\
(\mathrm{nm})\end{array}$ & $70-150$ & $50-120$ & $30-80$ & $20-60$ & $15-45$ & $10-30$ \\
\hline Surface concentration $\left(\mathrm{cm}^{-3}\right)$ & $10^{18}$ & $10^{18}$ & $10^{19}$ & $10^{19}$ & $10^{20}$ & $10^{20}$ \\
\hline Wafer Diameter $(\mathrm{mm})$ & 200 & 200 & 300 & 300 & 400 & 400 \\
\hline Metal Impurity $\left(\mathrm{at} / \mathrm{cm}^{2}\right)$ & $5 \times 10^{10}$ & $2.5 \times 10^{10}$ & $1.10^{10}$ & $5 \times 10^{9}$ & $2.5 \times 10^{9}$ & $<2.5 \times 10^{9}$ \\
\hline
\end{tabular}

\section{SHALLOW PROFILES, DEFECTS AND DOPANTS}

The reduction in device dimensions as seen in Table I leads to ever tighter requirements on the control of dopant profiles. With characteristic feature sizes now at a few tenths of micrometers this means both lateral and depth profiles must be controlled to the tens of nanometers. Since dopants are introduced by ion implantation this requires the resultant profiles to be known to such dimensions. Further, circuit designers utilizing TCAD tools wish to have this accuracy in their predictive models.

The as-implanted ion profiles as determined by SIMS depth profile measurements are typically modeled by Dual-Pearson multiparameter fits which have the necessary accuracy but lack predictability outside their range of fitting. This limitation is being addressed with increasing accuracy through Monte Carlo simulations with realistic physical-based models.[3] In Table II the key parameters for describing as-implanted profiles are indicated. At present accurate predictive descriptions of 2 and 3 dimensional effects and the accumulation of disorder (which can affect the as-implanted profiles through the altering of channeled ion trajectories) are the major challenges. In Fig. 2 an example of the Monte Carlo simulation of the 2D profile for boron is compared to a modified Pearson fit to the simulation result. In the case of 2 and 3D effects we are currently limited by the ability to readily measure such profiles at the needed tens of nm resolution. Thus calibration of $1 D$ profile modeling through SIMS is used to gain confidence in the predictive capability of the simulations and then the calculations are extended to 2 and $3 \mathrm{D}$ descriptions.

For the shallow implants used today for doping the source and drain the implantationinduced damage significantly affects the final doping profile. This effect occurs after the thermal 


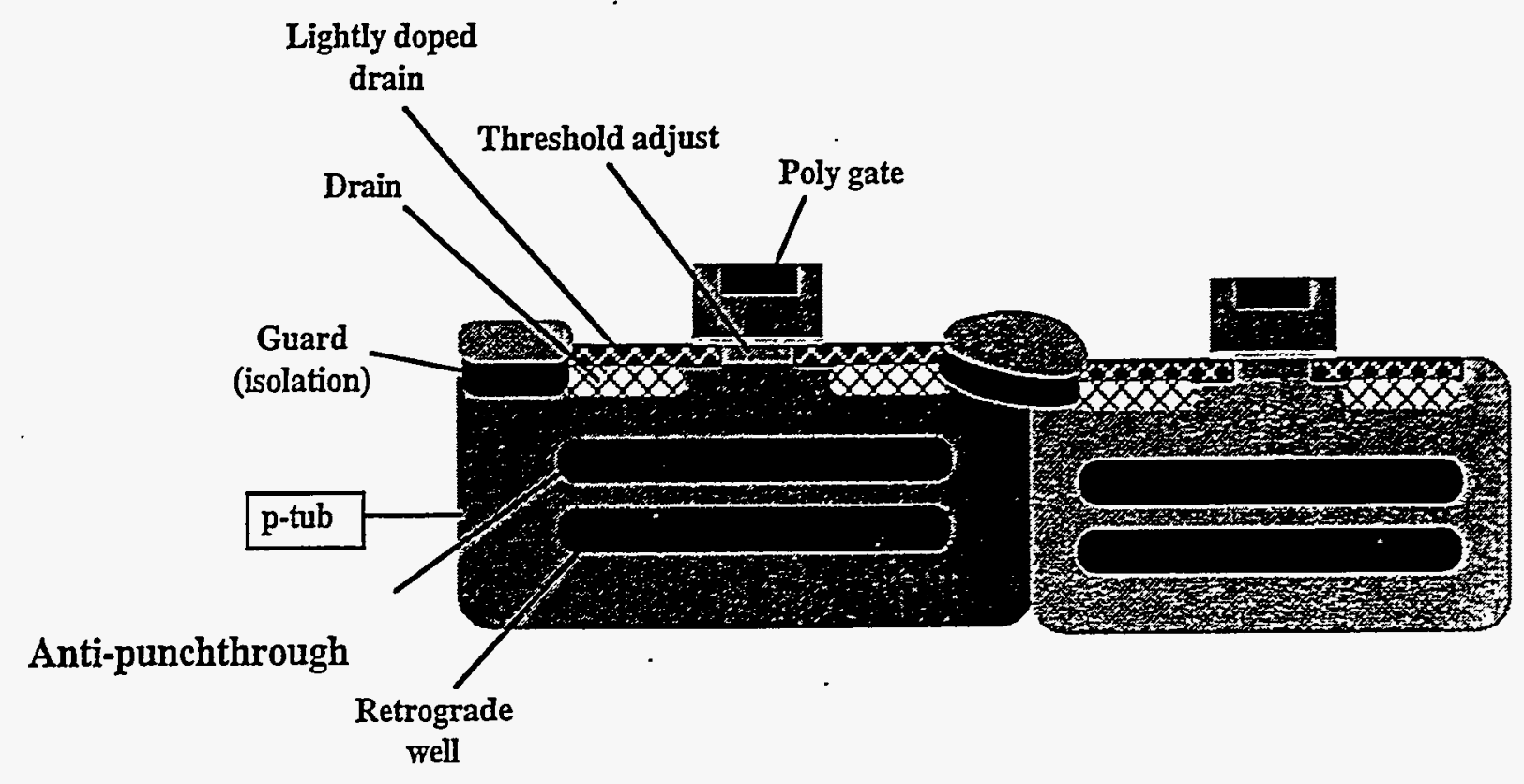

Figure 1. Schematic of complementary metal oxide semiconductor technology (CMOS) illustrating uses of ion implantation in semiconductor processing.

Table II. Parameters required for precise description of implant profiles.
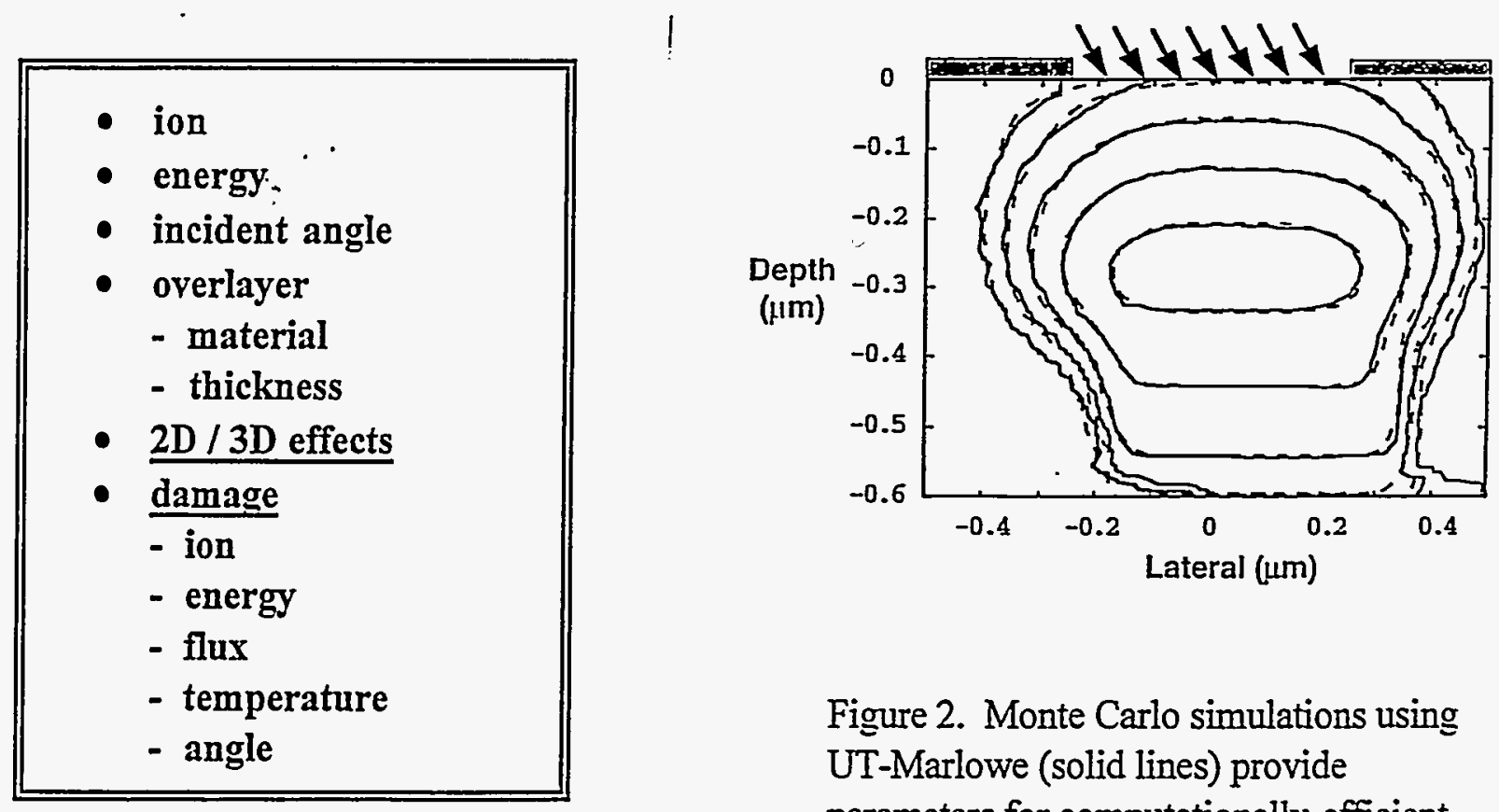

Figure 2. Monte Carlo simulations using UT-Marlowe (solid lines) provide parameters for computationally-efficient, modified Pearson models of 2-D implant profiles (dashed lines). Simulated implant conditions are for $80 \mathrm{keV} \mathrm{B}, 1 \times 10^{13} / \mathrm{cm}^{2}$, $10^{\circ}$ tilt. 
processing to activate the dopant and is due to defect-enhanced diffusion. It can extend and widen profiles by up to $100 \mathrm{~nm}$. While previously not of significant concern it has become a major issue at current device dimensions, leading to the unwanted coupling of performance to exact processing conditions through such device designer headaches as the reverse channel effect. The process is commonly referred to as transient enhanced diffusion and has stimulated a much closer examination of the defect production, storage, release and dopant-defect interactions than previously in the attempt to develop a microscopic understanding of the phenomena involved.

Theoretical studies of the damage production are increasingly using molecular dynamics simulations. The $\mathrm{MD}$ approach allows more accurate descriptions of the displacement cascade and resulting configuration of the atomic disorder than possible with the binary collision approximation. In Fig. 3 an example is shown of the $M D$ results for the evolution of a damage cascade for a $5 \mathrm{keV}$ Si recoil in Si.[4] While ion cascade disorder can be well treated the timescales for the subsequent defect interactions, diffusion and annealing are beyond the current computational power for $\mathrm{MD}$ and require kinetic Monte Carlo methods. Recent advances in large scale $a b$ initio calculations of stable configurations and minimum energy paths offers the possibility of defining much more accurate rules for the MC description of the diffusion, clustering, recombination, dissolution, and related events, offering the possibility of physically reasonable descriptions of the damage evolution. Such treatments will be critical to an eventual fully predictive modeling of the transient enhanced diffusion process.[5]

The ion damage provides a source term which leads to the clustering of defects. Upon higher temperature annealing these clusters provide a source of point defects for subsequent interstitial or vacancy enhanced migration of the implanted dopant atoms.[6] A schematic illustration of the process is given in Fig. 4 for the case of interstitials. This process provides the basis for transient enhanced diffusion (TED). For example for $B$, defect clusters such as the $\{311\}$ defects are believed to release interstitials, enhancing the $B$ migration via a kickout mechanism that.leads to an initial rapid migration of the $B$ in the 600 to $800^{\circ} \mathrm{C}$ annealing regime after which the $\mathrm{Si}$ interstitials are consumed and subsequent $\mathrm{B}$ migration occurs by normal thermally activated diffusion. Recently, the incorporation of sharp profiles of dopants by delta doping in MBE followed by Si overlayer growth and subsequent ion damage introduction at shallower depths has allowed the damage source terms and subsequent diffusion to be separated. This approach is illustrated in Fig. 5 for $\mathrm{B}$ in $\mathrm{Si}$. [7] By separate quantitative TEM measurements of the defect source terms, for example by observing \{311\} dissolution or dislocation loop growth or shrinkage, one can achieve a much more quantitative understanding of the processes involved in TED. This area is currently undergoing rapid advance and additional factors such as defectimpurity interactions with, for example, carbon are being identified. The fundamental barrier to a full description has been our lack of a detailed understanding of defect interactions and evolution in $\mathrm{Si}$, even though there have been many good basic studies and progress on the simpler defects in early studies. Here too, recent advances in ab initio calculations can play a role in identifying the fundamental dopant defect configurations and migration paths and energies.

\section{HIGHER ENERGY IMPLANTS AND GETTERING}

Another area of recent advance in both the science and technology for ion beam processing of semiconductors involves the use of higher energy ions. The main application is for forming the $\mathrm{n}$ and $\mathrm{p}$ type wells for CMOS circuits directly by implantation and activation anneals, rather than 
by shallow implants followed by long drive-in diffusions. Advantages include improved isolation, improved resistance to latch-up, reduction in the long drive-in anneals, and, most importantly, for sub $0.5 \mu \mathrm{m}$ CMOS a reduction of about $10 \%$ in the number of processing steps.[8]

One of the major issues in high energy implants is the formation of extended defects in active regions of devices between the ion end-of-range and the surface. Two interesting defect regimes are emerging. At the lower doses, typical of well formation, the dislocations that thread to the surface are of the interstitial type but their concentrations are insignificant for the doses now used for retrograde well formation. For the higher doses used in such applications as siliconon-insulator (SOI) formation the role of vacancy agglomeration and extended defect formation is now becoming evident. Such effects are illustrated in Fig. 6 for studies related to SOI oxygen implantation.[9] Although defect clusters are not visible by channeling or by TEM (left micrograph) in the region above the oxygen implant a small additional fluence leads to extended defects (middle micrograph). However clearly microscopic defect clusters are present below this threshold fluence because annealing of the lower fluence sample initially reveals small vacancy voids, and further, an additional implant and anneal before introducing the final fluence (referred to as a two-step implant) does not result in extended defects. In these higher energy implant cases most of the point defects in the region between the surface and the main end-of-range damage recombine. However the results again suggest the detailed nature of the microscopic defect storage in clusters and their subsequent accumulation and interaction are critical to a physically-based predictive capability. In Fig. 7 we illustrate schematically this evolution for vacancy-like defects. For vacancies we know more about simpler defects (vacancies, divacancies, 4-vacancies and vacancy-oxygen centers for example) but the picture is still far from complete. Understanding the full pathology of point defect formation, agglomeration and release is now becoming increasingly important in many aspects of Si science and technology.

Gettering of transition metal impurities by internal oxide precipitates is generally utilized in processing to minimize gate oxide degradation, minority carrier lifetime degradation, generationrecombination centers, and junction leakage. However the push to smaller device sizes and larger die (chip) sizes is leading to increasingly stringent limits on allowable metal impurities in the active device area (see Table I). As a result there has been a return of interest to exploring ion implantation for introducing gettering sites. In general the damage induced by implantation does not produce sufficiently strong or stable sites for gettering. However, recent studies with species like boron, carbon and He have raised the possibility of additional mechanisms for gettering impurities. For higher boron fluences for Fe impurity, for example, pairing occurs along with a Fermi level enhancement which can lead to significant trapping. Another interesting mechanism is found for the case of He implantation which forms nanocavities upon annealing.[10] Trapping of $\mathrm{Cu}$ for this case is illustrated in Fig. 8. The highly reactive internal surface of Si leads to segregation of a wide range of metal impurities in $\mathrm{Si}$. Perhaps even more significantly, these studies are leading the way to a more quantitative, physically-based modeling of gettering which will be enabled through such studies.

\section{CHARACTERIZATION AND MACHINE PERSPECTIVES}

The major challenge for characterization is the development of techniques to profile dopants in 2 dimensions. SIMS has the necessary sensitivity and resolution to determine dopantspecific profiles in depth.[11] But extending this technique to obtain 2D profiles does not appear 

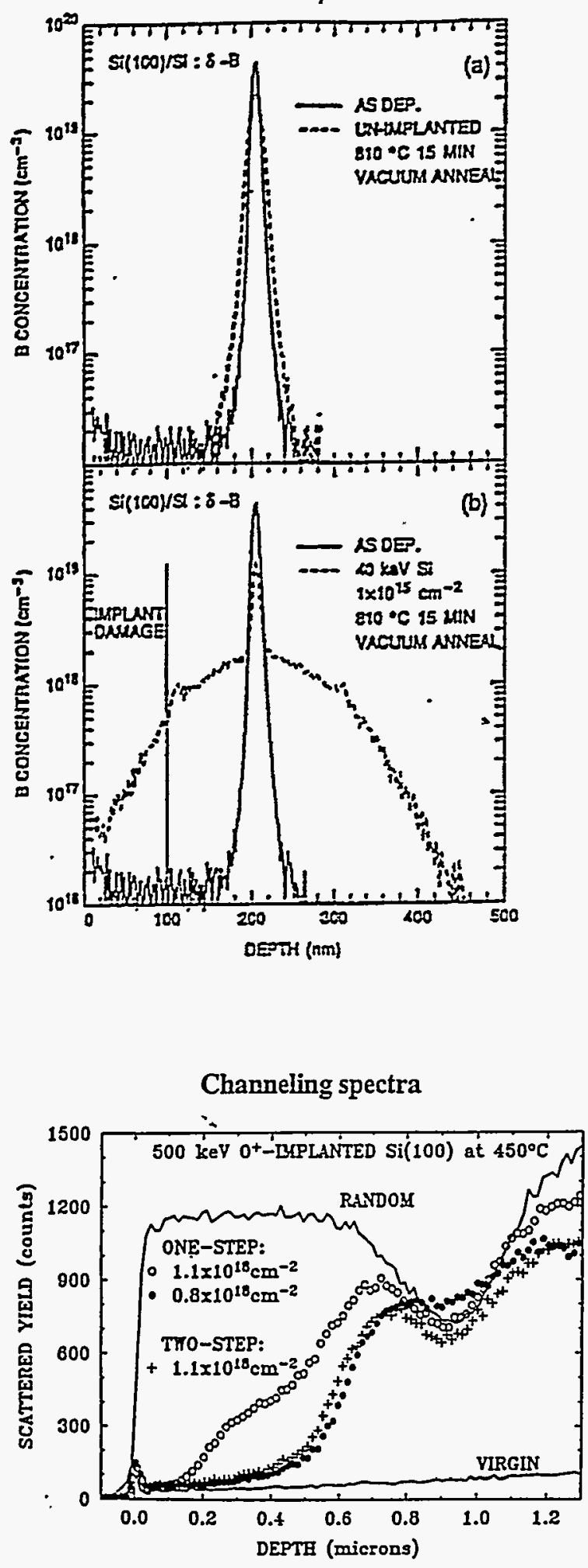

Figure 5. Ion-induced damage leads to transient enhanced diffusion of dopant profiles. (a) SIMS measurements of a B doping spike in MBE grown Si layers before and after diffusion at $810^{\circ} \mathrm{C}$ for $15 \mathrm{~min}$. (b) Same as (a) but with the B marker layer implanted with $1 \times 10^{15} / \mathrm{cm}^{2}, 40 \mathrm{keV} \mathrm{Si}$ before diffusion.

Figure 6. RBS spectra and XTEM micrographs from $\mathrm{Si}(100)$ implanted at $450^{\circ} \mathrm{C}$ with $500 \mathrm{keV}$ $\mathrm{O}^{+}$-ions. The spectra implanted continuously in a "single-step" (represented by open ( 0 ) and closed $(\bullet)$ circles) show a strong fluence dependence of damage creation; the "two-step" process involves interrupting the $\mathrm{O}^{+}$-implantation with a Si implant to create $\mathrm{Si}$ interstitials and annihilate vacancy-like defects. The XTEM images correspond to fluences of (a) $0.8 \times 10^{18}$ and (b) $1.1 \times 10^{13} \mathrm{~cm}^{-2}$. The micrograph in (c) shows the evolution of the damage morphology after annealing at $900^{\circ} \mathrm{C}$ of the $0.8 \times 10^{18} \mathrm{~cm}^{-2}$ implanted sample. 


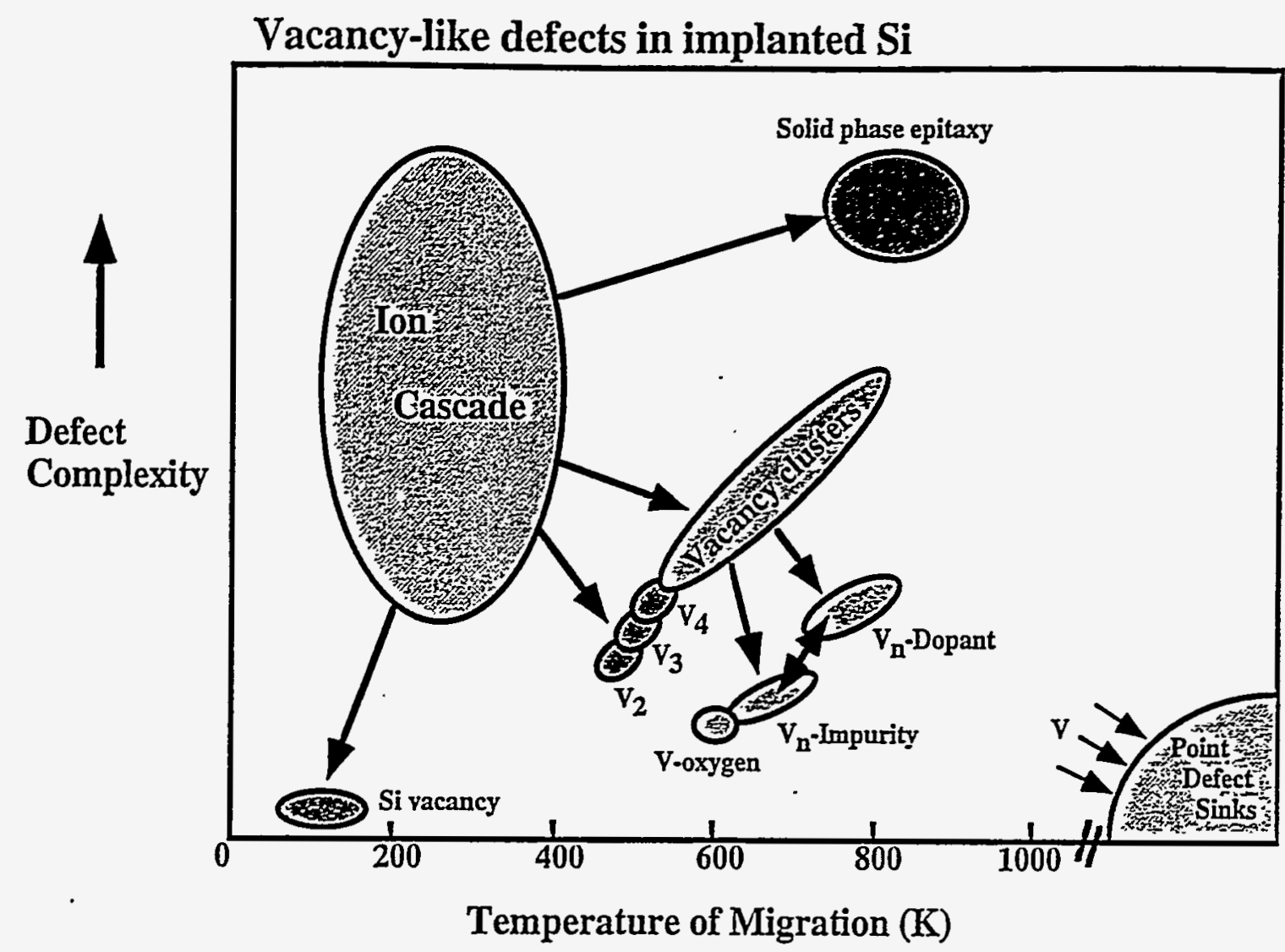

Figure 7. Comparison of the relative complexity of vacancy-like defects and defect clusters formed by high energy ions, and the temperatures at which these defects are active.
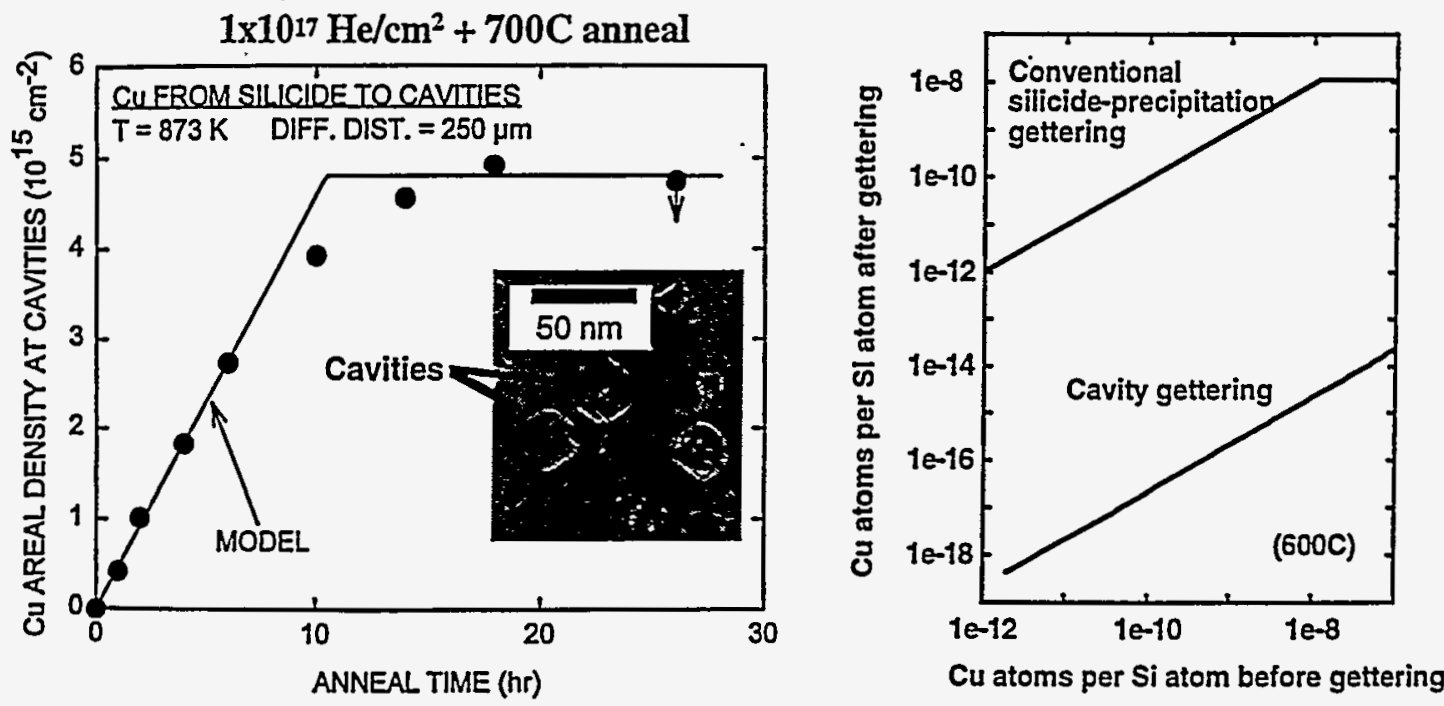

Figure 8. Ion-implanted nanocavities in Si are being studied for impurity gettering. (a) Diffusion of $\mathrm{Cu}$ from the implanted silicide to the cavities enables determination of the relative energies for silicide formation and trapping at internal cavity surfaces. (b) Gettering of $\mathrm{Cu}$ by nanocavities is predicted to extend many orders of magnitude lower than conventional silicide precipitation at low temperatures. 
possible for the desired $10 \mathrm{~nm}$ resolutions at dopant concentrations of $10^{18}$ to $10^{20} / \mathrm{cm}^{3}$ due to the small number of atoms involved ( 1 atom per $10 \mathrm{~nm}$ cube for $10^{18} / \mathrm{cm}^{3}$ ). Other approaches being explored include scanning capacitance and etching or staining in combination with electron or atomic force microscopies. For the sensitive detection of impurities recent advances in ion beam analysis techniques using heavy ion backscattering at low energies appears to be adequate for the low levels of metals of concern in gettering control.[12]

One of the major issues in ion implantation machines is the performance of implanters at low energies and high currents for shallow source and drain doping.[13] At present this corresponds to implants at 5 to $10 \mathrm{keV}$ for boron. Issues in reaching these lower energies include space-charge balance, transport, uniformity, surface charging, and beam divergence for tight lateral junction control. Machine designs have become quite sophisticated in their ability to ensure a very high degree of compensation of the ions by electrons along the beam path and also very complete charge neutralization at the wafer surface.

A variety of ion beam technologies continue to have more exploratory roles in semiconductor processing. Perhaps one of the most interesting possibilities would be a technique to simultaneously pattern and dope. While this is possible in principle with focused ion beams it is not practical due to the slow, linear nature of writing by scanning beams. The use of focused ion beams at present is primarily restricted to mask repair, rewiring in circuit development and device sectioning for failure analysis. Other approaches to patterning, such as projection ion lithography, and to patterned doping, such as gas immersion laser doping, are being explored. Plasma immersion implantation, as well as gas immersion laser doping, are interesting for their potential to provide a very shallow introduction of dopants.

\section{SUMMARY}

In summary there are still a large number of challenges in ion implantation processes, being driven primarily by the continual scaling of semiconductor processing technology to ever smaller dimensions. A major objective is to obtain a sufficiently detailed microscopic understanding of the processes involved so that accurate and truly predictive TCAD models can be developed for use by designers. A major outcome is the current push to understand defect processes in ion implanted $\mathrm{Si}$ in much greater detail than previously possible. Significant advances are possible by combining more quantitative experiments with newly available powerful computational tools. As a result we see a renaissance in the understanding of defect interactions in $\mathrm{Si}$.

\section{ACKNOWLEDGMENTS}

The discussions, support and encouragement of C. P. Flynn and J. Smith are gratefully acknowledged. This work supported by the U.S. Department of Energy through the Council on Materials and Basic Energy Sciences. Work at Sandia National Laboratories supported under Contract DE-AC04-94AL85000.

\section{REFERENCES}

1. The National Technology Roadmap for Semiconductors, (Semiconductor Industry Association, San Jose, 1994). 
2. The Panel, consisting of the authors of this manuscript, met in a Workshop format in Santa Fe, NM on May 4-7, 1995 to evaluate the status of the field and to assess critical opportunities and future challenges. The Panel was chaired by S. T. Picraux and J. M. Poate. A full report of the Panel is being coordinated by E. Chason and will appear in J. Appl. Phys. Reviews.

3. A.F. Tasch, S.H. Yang and S.J. Morris, Nucl. Instr. and Meth. B102, 173 (1995).

4. M.J. Caturla, T. Diaz de la Rubia and G.H. Gilmer, Nucl. Instr. and Meth. B106, 1 (1995).

5. M. Jaraiz, G.H. Gilmer, J.M. Poate and T. Diaz de la Rubia, App. Phys. Lett. 68, 409 (1996).

6. P.M. Fahey, P.B. Griffin and J.D. Plummer, Rev. Mod. Phys. 61, 289 (1989).

7. D.J. Eaglesham, P.A. Stolk, H.-J. Gossman, T.E. Haynes and J.M. Poate, Nucl. Instr. and Meth. B106, 191 (1995).

8. K. Tsukamoto, S. Kumori, T. Kuroi and Y. Akasaka, Nucl. Instr. and Meth. B59, 584 (1991).

9. D.S. Zhou, O.W. Holland and J.D. Budai, Appl. Phys. Lett. 63, 3580 (1993).

10. S.M. Myers, D.M. Follstaedt, G.A. Petersen, C.H. Seager, H.J. Stein and W.R. Wampler, Nuclear Instrum. and Meth. B106, 379 (1995).

11. C.W. Magee.and R.E. Honig, Surface and Interface Analysis 4, 36 (1982).

12. J.A. Knapp, J.C. Banks and B.L. Doyle, Nucl. Instrum. and Meth. B85, 120 (1994).

13. M.I. Current, R. Mathur, M. Kump and L.A. Larson, Nucl. Instrum. and Meth. B102, 198, (1995).

\section{DISCLAIMER}

This report was prepared as an account of work sponsored by an agency of the United States Government. Neither the United States Government nor any agency thereof, nor any of their employees, makes any warranty, express or implied, or assumes any legal liability or responsibility for the accuracy, completeness, or usefulness of any information, apparatus, product, or process disclosed, or represents that its use would not infringe privately owned rights. Referprocess disco trade name, trademark, manufacturer, or otherwise does not necessarily constitute or imply its endorsement, recommenter United States Government or any agency thereof. The views and opinions of authors expressed herein do not necessarily state or reflect those of the United States Government or any agency thereof. 